\title{
Comparison of outcomes of tyrosine kinase inhibitor in first- or second-line therapy for advanced non-small-cell lung cancer patients with sensitive EGFR mutations
}

\author{
Jianlin Xu ${ }^{1,}{ }^{*}$, Xueyan Zhang ${ }^{1, *}$, Haitang Yang ${ }^{2}$, Guozheng Ding ${ }^{3}$, Bo Jin ${ }^{1}$, Yuqing Lou ${ }^{1}$, \\ Yanwei Zhang ${ }^{1}$, Huimin Wang ${ }^{1}$, Baohui Han ${ }^{1}$ \\ ${ }^{1}$ Department of Pulmonary, Shanghai Chest Hospital, Shanghai Jiaotong University, Shanghai, China \\ ${ }^{2}$ Department of Thoracic Surgery, Shanghai Chest Hospital, Shanghai Jiaotong University, Shanghai, China \\ ${ }^{3}$ Department of Pulmonary, Anqing Municipal Hospital, Anhui, China \\ *These authors contributed equally to this work
}

Correspondence to: Baohui Han, email: xkyyhan@gmail.com

Keywords: EGFR TKI, NSCLC, first-line, second-line

Received: June 17, $2016 \quad$ Accepted: September 05, 2016

Published: September 15, 2016

\section{ABSTRACT}

Direct comparisons between the use of first- and second-line EGFR tyrosine kinase inhibitor (TKI) in patients with sensitive EGFR mutations are limited. A total of 264 advanced non-small-cell lung cancer (NSCLC) patients with sensitive mutations received EGFR TKI therapy as the first-line therapy, and a total of 187 patients received TKI as the second-line therapy at Shanghai Chest Hospital. First-line EGFR TKI therapy [12.9 months, 95\% confidence interval (CI), 10.7-15.2] provided longer progressionfree survival (PFS) than did second-line EGFR TKI therapy (9.0 months, 95\% CI, 7.7-10.2) [hazard ratio (HR): $0.78, P=0.034$ ]. The objective response rate (ORR) of first-, and second-line TKI therapy were $67.8 \%(159 / 233)$ and $55.6 \%(94 / 169)$, respectively $(P=0.001)$. The overall survival $(O S)$ for patients $(n=141)$ receiving first-line TKI followed by second-line chemotherapy were longer than those for patients $(n=187)$ receiving first-line chemotherapy followed by second-line TKI (HR: 0.69, $P=0.02$ ).

Compared with second-line TKI, first-line therapy achieved a significant and longer PFS, and higher ORR in the sensitive EGFR mutated NSCLC patients. The therapeutic strategy of using TKI followed by chemotherapy achieved longer OS than that using chemotherapy followed by TKI.

\section{INTRODUCTION}

Worldwide, lung cancer is the most frequently diagnosed cancer. Non-small-cell lung cancer (NSCLC) constitutes approximately $85 \%-90 \%$ of all lung cancers $[1,2]$. Platinum-based chemotherapy provides a survival benefit for patients with advanced lung cancer; however, most patients cannot survive more than 1 year [3].

The recognition of a subgroup of patients with NSCLC harboring mutations of EGFR that exhibit a favorable response to tyrosine kinase inhibitor (TKI) has changed the treatment patterns and outcomes of NSCLC [4-6]. Several randomized studies demonstrated that, for EGFR mutated NSCLC, first-line TKI therapy could provide higher tumor response rates $(\mathrm{RR})$ and longer progression-free survival (PFS) than chemotherapy. However, most of these studies failed to demonstrate improvement in overall survival (OS) [7-12]. The failure might be explained by the high proportion of patients from the chemotherapy arm crossing over to the EGFR TKI arm on progression $[13,14]$. Patients with EGFR mutations may also benefit from second- or third-line EGFR TKI therapy. Therefore, this raises the question of whether TKI is more effective in EGFR mutated NSCLC patients as a first-line therapy or is equally effective when administered as a second-line therapy [15].

In this study, we summarized the clinical data from patients at Shanghai Chest Hospital in order to compare the outcomes from TKIs used in first- or second-line therapy for advanced NSCLC patients with sensitive EGFR mutations. 


\section{RESULTS}

A total of 457 patients with sensitive EGFR mutations (19del or 21L858R) who received TKI therapy were identified (264 patients received TKI and 193 received chemotherapy as the first-line therapy). Among patients treated with TKI as the first-line treatment, 141 received chemotherapy as the second-line therapy. Among patients treated with chemotherapy as the first-line treatment, 187 received TKI as the second-line therapy. Demographic data of patients receiving TKI as the first- or second-line therapy are shown in Table 1.

\section{Efficacy of treatments}

First-line EGFR TKI therapy (12.9 months, 95\% CI, 10.7-15.2) provided a longer PFS than did secondline EGFR TKI therapy (9.0 months, 95\% CI, 7.7-10.2; HR, 0.78, 95\% CI, 0.61-0.98; $P=0.034$ ) (Figure 1). There were 402 patients available for an analysis of best response rate from TKI therapy. The overall response rates (ORRs) of first- and second-line TKI therapy for sensitive EGFR mutated patients were $67.8 \%(159 / 233)$, and $55.6 \%$ (94/169), respectively $(P=0.001)$ (Table 2$)$.

The OS analyses were conducted comparing two defined groups of patients: patients treated in sequence with first-line chemotherapy followed by TKI in the second-line (CT-TKI) $(n=187)$, and patients treated with first-line TKI followed by chemotherapy in the secondline (TKI-CT) $(n=141)$. A comparison of baseline characteristic of CT-TKI and TKI-CT cohorts are shown in Supplementary Table S1 and Supplementary Table S2. Our results demonstrated that the OS for the TKI-CT group (30.7 months, 95\% CI, 28.4-32.9) was longer than those in the CT-TKI group (27.2 months, 95\% CI, 24.8-29.6) (HR, 0.69, 95\% CI, 0.50-0.94; $P=0.02$ ) (Figure 2).

\section{DISCUSSION}

This retrospective analysis was performed in order to compare outcomes from EGFR TKI as first- or secondline therapy for advanced NSCLC patients with sensitive EGFR mutations. The results demonstrated that first-line TKI therapy provided superior RR and PFS than secondline TKI therapy, and patients receiving first-line TKI followed by second-line chemotherapy experienced a longer OS than those who received first-line chemotherapy followed by second-line TKI therapy.

Several randomized clinical trials have been used to evaluate first-line TKI therapy for EGFR mutated NSCLC [7-12]. Current guidelines suggest first-line TKI therapy for this population, based on the results of superior PFS and RR of first-line TKI, compared to chemotherapy [16]. However, most of these studies failed to demonstrate improvement in OS. This raises the question of whether TKI is more effective in EGFR mutated NSCLC as a first-line therapy or is equally effective when administered as a second-line therapy [15]. In the IPASS, WJTOG 3405, NEJ002, and OPTIMAL (CTONG-0802) studies, the ORR of first-TKI therapies were $64.3 \%, 62.1 \%, 73.7 \%$, and $82.9 \%$, respectively $[7,8,10,11]$. Due to limited sample size, the results of ORR for second-line TKI therapy for EGFR mutated NSCLC in previous clinical trials differed greatly. Nineteen EGFR mutated patients in the INTEREST study achieved an ORR of 42.1\% [17]. Twenty-six EGFR mutated patients from the ISEL study showed a tumor response rate of $37.5 \%$ [18]. A metaanalysis summarized TKIs as different line therapies for NSCLC patients with EGFR mutations, and the results demonstrated that the response rate was $70 \%$ in first-line trials, while in three second-line trials the response rate was $47.4 \%$ [19]. In the present study, first-line TKI therapy achieved an ORR of $67.8 \%$, which was significantly better than that achieved by second-line TKI therapy (55.6\%). Similar results were reported in the NEJ002 study, which demonstrated that the response rate of EGFR-TKI decreased from $73.3 \%$ with first-line treatment to $58.5 \%$ in second-line treatment [7]. The median PFS of firstline TKI therapy in the current study was consistent with previous reports [20]. Compared with second-line, firstline TKI therapy provided longer PFS. Similar results were achieved in a combined survival analysis, which demonstrated that first-line TKI therapy provided a better PFS than second- or third-line TKI therapy $(\mathrm{HR}=0.57$; $P=0.007$ ) [21]. Previously, a preclinical study used EGFR mutant NSCLC cell lines to explore whether prior exposure to platinum agents would affect subsequent responses to TKI therapy. The results suggested that firstline chemotherapy might reduce the benefit of subsequent EGFR-TKI treatment [22].

The high crossover rate to second-line or third-line EGFR TKI therapy in the first-line chemotherapy cohort could explain the failure to achieve a statistically longer OS in the first-line EGFR TKI therapy cohort. The PFS benefit of first-line TKIs did not appear to translate into an OS benefit in previous clinical trials. This could be partly explained by the subsequent effect of EGFR TKI therapy on OS. In the WJTOG 3405 study, more than $90 \%$ of the patients from the chemotherapy arm received EGFR TKI as a salvage therapy [13]. In the EURTAC study, the HR of OS for the first-line erlotinib arm versus the firstline chemotherapy arm was 0.92 (95\% CI, 0.63-1.35). However, after using statistical models to control for second-line post-study treatment effects, the HR for OS was 0.68 (95\% CI, 0.37-1.25) [14]. Similarly, the OPTIMAL study final OS results demonstrated that the median OS between the first-line erlotinib arm and the first-line chemotherapy arm was similar [23]. According to the in-depth analysis of the OPTIMAL study, $36.6 \%$ of the patients with common mutations that received first-line erlotinib did not receive post-study therapy, and $22.2 \%$ of the patients who received chemotherapy 
Table 1: Demographic data of patients receiving TKI as first-, or second-line therapy

\begin{tabular}{lccc}
\hline \multicolumn{1}{c}{ Characteristic } & First-line TKI $(\boldsymbol{n}=\mathbf{2 6 4})$ & Second-line TKI $(\boldsymbol{n}=\mathbf{1 8 7})$ & $\boldsymbol{P}$ \\
\hline Median age (range) & $63(32-86)$ & $61(30-81)$ & 0.134 \\
$\geq 60$ & $164(62.1 \%)$ & $103(55.1 \%)$ & \\
$<60$ & $100(37.9 \%)$ & $84(44.9 \%)$ & 0.210 \\
Gender & $103(39.0 \%)$ & $84(44.9 \%)$ & \\
Male & $161(61.0 \%)$ & $103(55.1 \%)$ & 0.602 \\
Female & & & \\
Smoking status & $58(22.0 \%)$ & $45(24.1 \%)$ & 0.003 \\
Smoker & $206(78.0 \%)$ & $142(75.9 \%)$ & \\
Never-smoker & & $161(86.1 \%)$ & 0.031 \\
Histology & $249(94.3 \%)$ & $26(13.9 \%)$ & \\
Adeno & $15(5.7 \%)$ & $38(20.3 \%)$ & \\
Others & & $95(50.8 \%)$ & \\
Types of EGFR TKI & $68(25.8 \%)$ & $54(28.9 \%)$ & 0.329 \\
Erlotinib & $147(55.7 \%)$ & & \\
Gefitinib & $49(18.6 \%)$ & $94(50.3 \%)$ & \\
Icotinib & & $93(49.7 \%)$ & \\
Mutation type & $145(54.9 \%)$ & $183(97.9 \%)$ & \\
19 del & $119(45.1 \%)$ & $4(2.1 \%)$ & \\
21 L858R & & & \\
PS & $242(91.7 \%)$ & & \\
$0-1$ & $18(6.8 \%)$ & & \\
$\geq 2$ & & & \\
\hline A & & & \\
\hline
\end{tabular}

Abbreviation: TKI, tyrosine kinase inhibitor.

did not receive any post-study treatment. This could partly explain why the first-line erlotinib arm did not show superiority in OS over the first-line chemotherapy arm. Unlike previous reports of the reversible EGFR TKIs, erlotinib and gefitinib, the combined analyses of LUX-Lung 3 and LUX-Lung 6 studies demonstrated that first-line irreversible TKI afatinib provided a longer OS for common EGFR mutated NSCLC patients compared with chemotherapy [24]. In those two studies, very few patients in the first-line chemotherapy cohorts received afatinib as a subsequent treatment, because afatinib was not clinically available at the time of the studies, so most of the patients received erlotinib or gefitinib after chemotherapy $[25,26]$. According to the results of the Lux Lung 7 study, afatinib

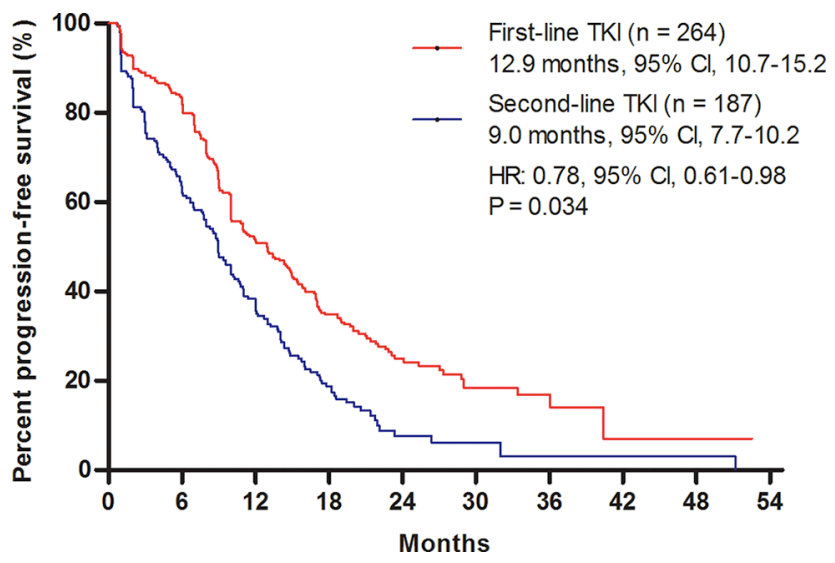

Figure 1: Comparison of progression-free survival (PFS). Kaplan-Meier survival curves for PFS analyses between first- and second-line TKI therapies. TKI, tyrosine kinase inhibitor. 
Table 2: Best response to EGFR TKI therapy

\begin{tabular}{lcc}
\hline \multicolumn{1}{c}{ Response rate (RR) } & First-line TKI therapy $(\boldsymbol{n}=\mathbf{2 3 3})$ & Second-line TKI therapy $(\boldsymbol{n}=\mathbf{1 6 9})$ \\
\hline Complete response (CR), $n(\%)$ & $11(4.7 \%)$ & $2(1.2 \%)$ \\
Partial response (PR), $n(\%)$ & $147(63.1 \%)$ & $92(54.4 \%)$ \\
Stable disease (SD), $n(\%)$ & $58(24.9 \%)$ & $55(30.8 \%)$ \\
Progressive disease (PD), $n(\%)$ & $17(7.3 \%)$ & $23(13.6 \%)$ \\
Objective response rate (ORR), $n(\%)$ & $158(67.8 \%)$ & $94(55.6 \%)$ \\
\hline
\end{tabular}

Abbreviation: TKI, tyrosine kinase inhibitor.

might provide a longer PFS compared with gefitinib in treatment-naive patients with EGFR-mutated NSCLC [27].

In the present study, the OS analyses were conducted between two defined groups of patients: patients treated in sequence with first-line chemotherapy, followed by TKI in the second-line (CT-TKI), and patients treated with firstline TKI, and chemotherapy in the second-line (TKI-CT). The OS for the TKI-CT group (30.7 months) was longer than that of the CT-TKI group (27.2 months) (HR, 0.69; $P=0.02$ ). These results were consistent with a previous study, which demonstrated that median OS in the gefitinib group was 7 months longer than that of the chemotherapy group (all patients were given gefitinib as the second-line treatment) [7]. Previously, the TORCH study compared first-line EGFR TKI followed by chemotherapy with first-line chemotherapy followed by second-line EGFR TKI. Subgroup analyses showed that patients with EGFR mutations experienced a greater benefit from first-line EGFR TKI followed by second-line chemotherapy [28]. The results of health-related quality of life $(\mathrm{QoL})$ studies in previous clinical trials also supported first-line TKI therapy. The QoL of patients receiving first-line EGFR TKI was better than that of patients receiving first-line chemotherapy $[7,11,29]$. In the results of the LUX lung 3 compared with cisplatin/pemetrexed, first-line afatinib prolonged the time of deterioration of cough and dyspnea symptoms [25]. Some experts suggested that if TKIs were administered as a second- or third-line treatment, patients might miss the best opportunity to receive treatment with TKIs, because of a rapidly progressive disease during or after the first-line treatment [15].

A major limitation of this study is its retrospective nature. We could not collect the data of patients' QoL in the present study. In addition, due to the limited database, the ORR and PFS of second-line chemotherapy could not be determined. We could not compare the efficacy of first-line and second-line chemotherapy in the present study. According to the NEJ002 study, prior EGFR TKI therapy would not influence the efficacy of subsequent chemotherapy in patients with EGFR mutant NSCLC [7]. However, another study demonstrated a reduced sensitivity of subsequent chemotherapy compared with that of TKInaïve frontline chemotherapy [30]. These results warrant more investigation.

In conclusion, compared with second-line TKI, first-line therapy achieved a longer PFS and a higher ORR in advanced NSCLC patients harboring sensitive EGFR mutations. The therapeutic strategy of using TKIs followed by chemotherapy can achieve longer OS than that using chemotherapy followed by TKIs, therefore this strategy should be considered as an optimal treatment option.

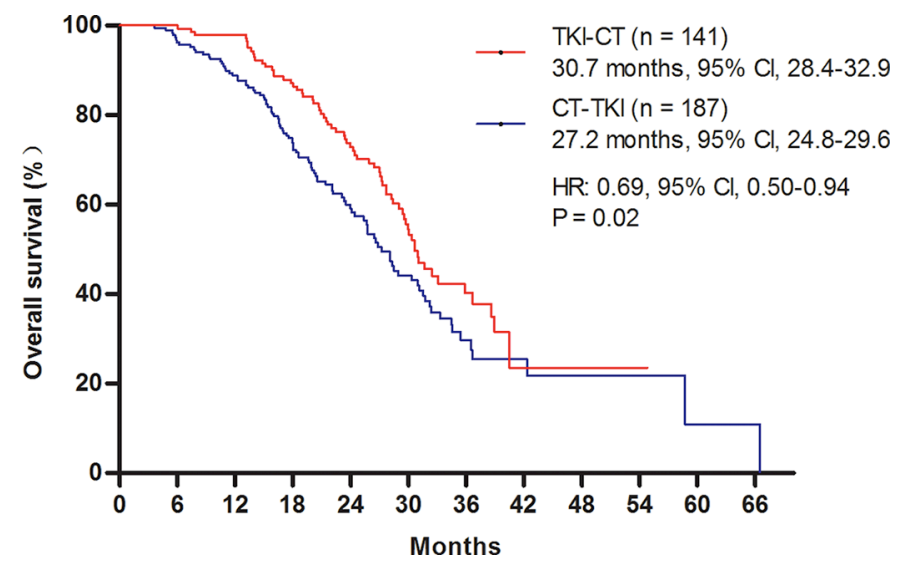

Figure 2: Comparison of overall survival (OS). Kaplan-Meier survival curves for OS analyses between first- and second-line TKI therapies. TKI, tyrosine kinase inhibitor. 


\section{MATERIALS AND METHODS}

\section{Study design and patients}

This study was designed to compare first- and second-line EGFR TKI therapies in patients with sensitive EGFR mutations. The study was approved by the Institutional Review Board of the Shanghai Chest Hospital. All of the patients were diagnosed with advanced NSCLC (stage IV) at the Shanghai Chest Hospital between January 2009 and December 2013. Baseline clinical characteristics included age at diagnosis, tumor histology, smoking history, and sex. We also abstracted treatment details, such as first- and second-line therapies, and the dates at which each line of therapy started. The inclusion criteria were (1) a pathologically confirmed advanced NSCLC, (2) a sensitive EGFR mutation consisting of an exon 19 deletion or an exon 21 point mutation (L858R), and (3) EGFR TKI therapy. Patients without survival and therapy details were excluded. The primary outcome was OS.

\section{Test method for EGFR mutations}

DNA was extracted from five serial slices of a $5-\mu \mathrm{m}$ paraffin section using the DNA FFPE Tissue Kit (Qiagen, Hilden, Germany). The highly sensitive method termed Amplification Refractory Mutation System (ARMS) was used to detect mutations in the EGFR gene according to the manufacturer's protocol of the DxS EGFR mutation test kit (DxS) [31].

\section{Statistical methods}

For descriptive purposes, demographic and clinical data are summarized as medians with ranges for continuous variables, and categorical variables are expressed as summarized by the means of absolute and percentage numbers. The associations between patient demographics were examined using Pearson's $\chi^{2}$ test. Survival results are summarized as median values and two-sided 95\% confidence intervals (CIs), and were analyzed using Kaplan-Meier analyses, whereas the logrank test was used for comparisons among subgroups. Multivariable adjusted hazard ratios (HRs) for allcause mortality by patient and treatment pattern were estimated using Cox regression. HRs were calculated along with their corresponding $95 \%$ CIs as measurements of association. Statistical significance was defined as $P<0.05$. SPSS software, version 22 (SPSS Inc., Chicago, IL, USA) was used for all statistical analyses.

\section{ACKNOWLEDGMENTS AND FUNDING}

This study was supported by the Key Projects of the Biomedicine Department, Science and Technology Commission of Shanghai Municipality (Project No.
11411951200). The funding agencies played no role in the study design, data collection and analysis, decision to publish, or preparation of the manuscript.

\section{CONFLICTS OF INTEREST}

The authors declare no conflicts of interest.

\section{REFERENCES}

1. Siegel R, Naishadham D, Jemal A. Cancer statistics, 2013. CA Cancer J Clin. 2013; 63:11-30.

2. Chen W, Zheng R, Zeng H, Zhang S, He J. Annual report on status of cancer in China, 2011. Chin J Cancer Res. 2015; 27:2-12.

3. Sacher AG, Le LW, Lau A, Earle CC, Leighl NB. RealWorld Chemotherapy Treatment Patterns in Metastatic Non-Small Cell Lung Cancer: Are Patients Undertreated? Cancer. 2015; 121:2562-9.

4. Lynch TJ, Bell DW, Sordella R, Gurubhagavatula S, Okimoto RA, Brannigan BW, Harris PL, Haserlat SM, Supko JG, Haluska FG, Louis DN, Christiani DC, Settleman J, et al. Activating mutations in the epidermal growth factor receptor underlying responsiveness of nonsmall cell lung cancer to gefitinib. New Engl J Med.2004; 350:2129-39.

5. Kris MG, Johnson BE, Berry LD, Kwiatkowski DJ, Iafrate AJ, Wistuba II, Varella-Garcia M, Franklin WA, Aronson SL, Su PF, Shyr Y, Camidge DR, Sequist LV, et al. Using multiplexed assays of oncogenic drivers in lung cancers to select targeted drugs. Jama. 2014; 311:1998-2006.

6. Russo A, Franchina T, Ricciardi GR, Picone A, Ferraro G, Zanghì M, Toscano G, Giordano A, Adamo V. A decade of EGFR inhibition in EGFR-mutated non small cell lung cancer (NSCLC): Old successes and future perspectives. Oncotarget. 2015; 6:26814-25. doi: 10.18632/ oncotarget.4254.

7. Maemondo $\mathrm{M}$, Inoue A, Kobayashi K, Sugawara S, Oizumi S, Isobe H, Gemma A, Harada M, Yoshizawa H, Kinoshita I, Fujita Y, Okinaga S, et al. Gefitinib or chemotherapy for non-small-cell lung cancer with mutated EGFR. N Engl J Med. 2010; 362:380-8.

8. Mitsudomi T, Morita S, Yatabe Y, Negoro S, Okamoto I, Tsurutani J, Seto T, Satouchi M, Tada H, Hirashima T, Asami K, Katakami N, Takada M, et al. Gefitinib versus cisplatin plus docetaxel in patients with non-small-cell lung cancer harbouring mutations of the epidermal growth factor recep-tor (WJTOG3405): an open label, randomised phase 3 trial. Lancet Oncol. 2010; 11:121-8.

9. Rosell R, Carcereny E, Gervais R, Vergnenegre A, Massuti B, Felip E, Palmero R, Garcia-Gomez R, Pallares C, Sanchez JM, Porta R, Cobo M, Garrido P, et al. Erlotinib versus standard che-motherapy as first-line treatment for European patients with advanced EGFR mutation-positive non-small-cell lung cancer (EURTAC): 
a multicentre, open-label, randomised phase 3 trial. Lancet Oncol. 2012; 13:239-46.

10. Mok TS, Wu YL, Thongprasert S, Yang CH, Chu DT, Saijo N, Sunpaweravong P, Han B, Margono B, Ichinose Y, Nishiwaki Y, Ohe Y, Yang JJ, et al. Gefitinib or carboplatinpaclitaxel in pulmonary adenocarcinoma. N Engl J Med. 2009; 361:947-57.

11. Zhou $\mathrm{C}, \mathrm{Wu}$ YL, Chen G, Feng J, Liu XQ, Wang C, Zhang S, Wang J, Zhou S, Ren S, Lu S, Zhang L, Hu C, et al. Erlotinib versus chemotherapy as first-line treatment for patients with advanced EGFR mutation-positive nonsmall-cell lung cancer (OPTIMAL, CTONG-0802): a multicentre, open-label, randomised, phase 3 study. Lancet Oncol. 2011; 12:735-42.

12. Soria JC, Wu YL, Nakagawa K, Kim SW, Yang JJ, Ahn MJ, Wang J, Yang JC, Lu Y, Atagi S, Ponce S, Lee DH, Liu Y, et al. Gefitinib plus chemotherapy versus placebo plus chemotherapy in EGFR-mutation-positive non-smallcell lung cancer after progression on first-line gefitinib (IMPRESS): a phase 3 randomised trial. Lancet Oncol. 2015; 16:990-8.

13. Mitsudomi T, Morita S, Yatabe Y, Negoro S, Okamoto I, Seto T, Satouchi M, Tada H, Hirashima T, Asami K, Katakami N, Takada M, Yoshioka H, et al. Updated overall survival results of WJTOG 3405, a randomized phase III trial comparing gefitinib $(G)$ with cisplatin plus docetaxel (CD) as the first-line treatment for patients with non-small cell lung cancer harboring mutations of the epidermal growth factor receptor (EGFR). J Clin Oncol. 30:485s, 2012; (suppl; abstr 7521).

14. Leon L, Golsorkhi A, Liu S, Drozdowskyj A, Rosell R. Overall survival analyses of first-line erlotinib versus chemotherapy in the EURTAC study population controlling for the use of post-study therapy. Eur Soc Med Oncol Meet, Madrid, Spain, 27 September, (Poster 1273).

15. Mok T, Yang JJ, Lam KC. Treating patients with EGFRsensitizing mutations: first line or second line-is there a difference? J Clin Oncol. 2013; 31:1081-8.

16. Masters GA, Temin S, Azzoli CG, Giaccone G, Baker S Jr, Brahmer JR, Ellis PM, Gajra A, Rackear N, Schiller JH, Smith TJ, Strawn JR, Trent D, et al. American Society of Clinical Oncology Clinical Practice. Systemic Therapy for Stage IV Non-Small-Cell Lung Cancer: American Society of Clinical Oncology Clinical Practice Guideline Update. J Clin Oncol. 2015; 33:3488-515

17. Douillard JY, Shepherd FA, Hirsh V, Mok T, Socinski MA, Gervais R, Liao ML, Bischoff H, Reck M, Sellers MV, Watkins CL, Speake G, Armour AA, et al. Molecular predictors of outcome with gefitinib and docetaxel in previously treated non-small-cell lung cancer: data from the randomized phase III INTEREST trial. J Clin Oncol. 2010; 28:2167-73

18. Taron M, Ichinose $\mathrm{Y}$, Rosell R, Mok T, Massuti B, Zamora L, Mate JL, Manegold C, Ono M, Queralt C,
Jahan T, Sanchez JJ, Sanchez-Ronco M, et al, Activating mutations in the tyrosine kinase domain of the epidermal growth factor receptor are associated with improved survival in gefitinib-treated chemorefractory lung adenocarcinomas. Clin Cancer Res. 2005; 11:5878-85.

19. Petrelli F, Borgonovo K, Cabiddu M, Barni S. Efficacy of EGFR tyrosine kinase inhibitors in patients with EGFRmutated non-small-cell lung cancer: a meta-analysis of 13 randomized trials. Clin Lung Cancer. 2012; 13:107-14.

20. Zhang Y, Sheng J, Yang Y, Fang W, Kang S, He Y, Hong S, Zhan J, Zhao Y, Xue C, Ma Y, Zhou T, Ma S, et al. Optimized selection of three major EGFR-TKIs in advanced EGFR-positive non-small cell lung cancer: a network metaanalysis. Oncotarget. 2016; doi: 10.18632/ oncotarget.7713. [Epub ahead of print].

21. Morita S, Okamoto I, Kobayashi K, Yamazaki K, Asahina H, Inoue A, Hagiwara K, Sunaga N, Yanagitani N, Hida $\mathrm{T}$, Yoshida $\mathrm{K}$, Hirashima $\mathrm{T}$, Yasumoto $\mathrm{K}$, et al. Combined Survival Analysis of Prospective Clinical Trials of Gefitinib for Non-Small Cell Lung Cancer with EGFR Mutations. Clin Cancer Res. 2009; 15:4493-8.

22. Chin TM, Quinlan MP, Singh A, Sequist LV, Lynch TJ, Haber DA, Sharma SV, Settleman J. Reduced erlotinib sensitivity of epidermal growth factor receptor-mutant non-small cell lung cancer following cisplatin exposure: A cell culture model of second-line erlotinib treatment. Clin Cancer Res. 2008; 14:6867-76.

23. Zhou C, Wu YL, Chen G, Feng J, Liu XQ, Wang C, Zhang S, Wang J, Zhou S, Ren S, Lu S, Zhang L, Hu C, et al. Final overall survival results from a randomised, Phase III study of erlotinib versus chemotherapy as first-line treatment of EGFR mutation-positive advanced non-smallcell lung cancer (OPTIMAL, CTONG-0802). Ann Oncol. 2015; 26:1877-83.

24. Yang JC, Wu YL, Schuler M, Sebastian M, Popat S, Yamamoto N, Zhou C, Hu CP, O’Byrne K, Feng J, Lu S, Huang Y, Geater SL, et al. Afatinib versus cisplatinbased chemotherapy for EGFR mutation-positive lung adenocarcinoma (LUX-Lung 3 and LUX-Lung 6): analysis of overall survival data from two randomised, phase 3 trials. Lancet Oncol. 2015; 16:141-51.

25. Yang JC, Hirsh V, Schuler M, Yamamoto N, O’Byrne KJ, Mok TS, Zazulina V, Shahidi M, Lungershausen J, Massey D, Palmer M, Sequist LV. Symptom control and quality of life in LUX-Lung 3: a phase III study of afatinib or cisplatin/pemetrexed in patients with advanced lung adenocarcinoma with EGFR mutations. J Clin Oncol. 2013; 31:3342-50.

26. Wu YL, Zhou C, Hu CP, Feng J, Lu S, Huang Y, Li W, Hou M, Shi JH, Lee KY, Xu CR, Massey D, Kim M, et al. Afatinib versus cisplatin plus gemcitabine for first-line treatment of Asian patients with advanced non-small-cell lung cancer harbouring EGFR mutations (LUX-Lung 6): an open-label, randomised phase 3 trial. Lancet Oncol. 2014; $15: 213-22$. 
27. Park K, Tan EH, O’Byrne K, Zhang L, Boyer M, Mok T, Hirsh V, Yang JC, Lee KH, Lu S, Shi Y, Kim SW, Laskin J, et al. Afatinib versus gefitinib as first-line treatment of patients with EGFR mutation-positive non-small-cell lung cancer (LUX-Lung 7): a phase 2B, open-label, randomised controlled trial. Lancet Oncol. 2016; 17:577-89.

28. Gridelli C, Ciardiello F, Gallo C, Feld R, Butts C, Gebbia V, Maione P, Morgillo F, Genestreti G, Favaretto A, Leighl N, Wierzbicki R, Cinieri S, et al. First-line erlotinib followed by second-line cisplatin-gemcitabine chemotherapy in advanced non-small-cell lung cancer: the TORCH randomized trial. J Clin Oncol. 2012; 30:3002-11.

29. Thongprasert S, Duffield E, Saijo N, Wu YL, Yang JC, Chu DT, Liao M, Chen YM, Kuo HP, Negoro S, Lam KC,
Armour A, Magill P, et al. Health-related quality-of-life in a randomized phase III first-line study of gefitinib versus carboplatin/paclitaxel in clinically selected patients from Asia with advanced NSCLC (IPASS). J Thorac Oncol. 2011; 6:1872-80.

30. Zeng Z, Yan HH, Zhang XC, Zhong WZ, He YY, Guan JL, Niu FY, Xie Z, Huang YS, Xu CR, Dong S, Wu YL. Reduced chemotherapy sensitivity in EGFR-mutant lung cancer patient with frontline EGFR tyrosine kinase inhibitor. Lung Cancer. 2014; 86:219-24.

31. Zhang Q, Zhu L, Zhang J. Epidermal growth factor receptor gene mutation status in pure squamous-cell lung cancer in Chinese patients. BMC Cancer. 2015; 15:88. 